\title{
Oui au bon sens, non à l'idéologie dogmatique
}

\footnotetext{
* Notre confrère Daniel Beutler, qui fait connaître son passé de dépendance, est membre du comité référendaire. Il n'est pas le seul parmi d'anciens toxicomanes qui, s'en étant sortis, deviennent les avocats de positions dures. Ces personnes et leurs parcours de vie toujours difficiles ont tout mon respect, mais elles ne sont pas majoritaires et on ne saurait baser une politique équilibrée, à l'intention de toute une collectivité, sur les convictions de quelques-uns fondées sur leur cas individuel. Plutôt, cela confirme le besoin d'assurer un éventail diversifié d'offres.
}

1 Romann C. Politique de la drogue: oui à une solution qui a fait ses preuves. Bull Méd Suisses. 2008; 89(37): 1569.

2 Hampton T. Abstinence-only programs under fire. JAMA. 2008;299. 2013-5.
Christine Romann, membre du Comité central, a complètement raison d'évoquer les «débats idéologiques ne menant à rien» [1]; j'en ai pour ma part trop vécu. Dans une carrière de santé publique où mon rôle incluait la prévention et la lutte contre la toxicomanie, en contact constant avec les confrères praticiens, cela a été une entreprise de longue haleine de faire prévaloir la raison, le pragmatisme. Avec des frustrations ... ces efforts n'ont pas toujours été couronnés de succès, à cause de l'influence en particulier de positions doctrinaires éloignées de la réalité, proches parfois du terrorisme intellectuel, de milieux s'improvisant experts alors que leurs buts sont typiquement idéologiques. Ces milieux s'opposent à l'actuelle révision de la LStup alors que, précisément, elle introduit des aménagements de bon sens, basés sur les faits scientifiques et empiriques, avec la nécessaire confirmation de la pertinence de la politique des quatre piliers.

L'espace ne permet pas d'aborder chacun de ses éléments (on se renseignera utilement sur le site www.lstup.ch). A noter un aspect qui concerne le corps médical: la littérature démontre que, dans des indications choisies, le cannabis apporte une vraie contribution à la lutte contre la douleur. La loi révisée permettrait de tels traitements mais les jusqu'auboutistes, adeptes de la funeste «War on Drugs» américaine, s'opposent à ce que ce progrès soit à la disposition des malades suisses ...

L'actualité fait évoquer un autre sujet. On s'émerveille de voir les contorsions des républicains américains devant les contradictions entre leurs positions rigoristes et les faits de la vie. Ainsi à propos de la grossesse d'une fille adolescente de Sarah Palin. L'abstinence avant mariage est un des axiomes que l'autoproclamée «Coalition chrétienne» aimerait imposer à ses concitoyens. Or, un récent article du JAMA [2] relève que les programmes d'abstinence à eux seuls n'ont «absolument aucun effet mesurable sur l'âge du premier rapport sexuel, le nombre de partenaires, les grossesses, les maladies sexuellement transmissibles». Occasion de rappeler que des études solides, au plan international, démontrent que les jeunes qui ont bénéficié d'une éducation sexuelle adéquate, non moralisante, ont des relations sexuelles moins précoces, moins fréquentes et moins à risque que ceux qui n'en ont pas bénéficié. Pour la drogue aussi, une information objective vaut mieux qu'une diabolisation à laquelle la jeunesse ne croit pas.

Je suis favorable à ce que tous ceux qui souffrent de dépendance (y compris à l'alcool, y compris au tabac) puissent s'en libérer, grâce à des aides adaptées à leur situation personnelle et de santé. L'expérience montre qu'il est impératif de disposer d'une diversité de méthodes, qui puissent l'une ou l'autre être proposées au bon moment (aucun professionnel ne peut penser aujourd'hui qu'il y aurait une «seule bonne méthode»)*. L'obsession doctrinaire de l'abstinence est aussi incapable à elle seule de résoudre les problèmes en matière de produits illégaux qu'elle l'est pour la sexualité des jeunes.

Ce n'est pas par des mesures «tunnelisées»sur l'abstinence et la répression qu'on gérera mieux la problématique de la drogue. Il n'est pas bon d'être aveugle à ce que nos sociétés ont vécu depuis 40 ans et il faut avoir l'intelligence et le courage politique d'en tenir compte. Les médecins doivent faire savoir que l'application de la politique impeccablement «vertueuse» que voudraient les opposants à la LStup aurait des conséquences tout à fait défavorables: punitives, rejetantes et pathogènes: plus de maladies infectieuses, plus de déchéance de ceux qui temporairement n'arrivent pas à s'astreindre à un régime exigeant, plus de délinquance. Voulonsnous cela? Il convient d'accepter la révision de la loi fédérale sur les stupéfiants.

Dr Jean Martin, ancien médecin cantonal vaudois et rédacteur du BMS 